\title{
Energy Efficient with Network Coding Multipath Routing Algorithm in Wireless Sensor Networks
}

\author{
Chao Gui ${ }^{1}$, Hua Chen ${ }^{2}$, Baolin Sun ${ }^{1}$ and Ying Song ${ }^{1}$ \\ ${ }^{1}$ School of Information and Management, Hubei University of Economics, Wuhan \\ 430205, Hubei Province, P. R. China \\ ${ }^{2}$ College of Mathematics \& Computer Science, Wuhan Textile University, Wuhan \\ 430065, Hubei Province, P. R. China \\ gui_chao@126.com,qiuchen_1022@163.com,blsun@163.com,prisong@163.com
}

\begin{abstract}
Network coding is a new paradigm in data transport and promises to change many aspects of wireless sensor network (WSN). Thanks to the recently developed network coding techniques, a high-throughput low-complexity hierarchical protocol can be facilitated due to the multiple-source relay-based data transmissions built on the network coding schemes, especially for the data communications between the source node and destination node. In this paper, we propose an Energy efficient that carefully couples Network coding and Multipath routing algorithm in Wireless Sensor Networks (ENM-WSN). Through an analytical study, we provide guidance on how to choose parameters in our scheme and demonstrate that the scheme is efficient in both multipath and energy consumption. We also present an algorithm for deciding the network coding scheme for a node to further reduce energy consumption by minimizing redundant packet transmissions. Simulation results show that, with the proposed energy efficient with network coding in WSN multipath routing protocol (ENM-WSN), energy consumption, packet delivery ratio, and network lifetime can be improved in most of cases. It is an available approach to multipath routing decision.
\end{abstract}

Keywords: WSN, energy efficient, network coding, multipath routing, performance evaluation

\section{Introduction}

A wireless sensor networks (WSN) consists of multiple nodes that maintain network connectivity through wireless communications. This connectivity is enabled via radio transmissions generated by a set of cooperating nodes [1-7]. In such networks, the nodes are often powered by battery, and energy efficient operations are critical to prolong the lifetime of the connections. Designing an energy efficient and reliable routing protocol for such networks is a challenging issue [7-12]. However, for real-time sensing, latency and reliability are of paramount importance, whereas in battery powered sensor networks, energy Efficient is an important metric.

Although multipath routing can increase reliability of transmission, too many paths may increase data redundancy and energy consumption. Multipath routing adopts parallel mechanisms to transmit data. Anantapalli et al. [5] have investigated the performance comparison among several multipath routing protocols in mobile ad hoc networks. Leinonen et al. [6] propose a distributed algorithm for solving the convex problem with partial dual decomposition approach by jointly optimizing the routing and the power allocation. Energy 
efficient protocol design deals with all layers of protocol stack and usually spans the network layer and MAC layer, in particular there is an increasing interest in algorithms for the network layer, namely energy efficient routing algorithms. Wang et al. [7] propose an energy efficient and collision aware (EECA) node-disjoint multipath routing algorithm. The data collection rate in pervasive healthcare systems is high. The development of efficient data and energy processing techniques are of great importance. One of the bottlenecks of sensor devices is the batteries. Considering the likelihood of forgetting to recharge the batteries of several sensors, this is a significant issue to be solved. Although there is much effort on designing low-power sensors to minimize this bottleneck [8], we still need energy scavenging techniques. Moreover, the data loss increases due to frequent network topology change and link error. Network coding technology adds a new mechanism to overcome these disadvantages of traditional multipath routing.

Network coding (NC) has emerged as a viable means to improve network throughput in WSN. Messages at the packet level are linearly combined at intermediate nodes and forwarded to multiple intended destinations. Since the initial proposal by Ahlswede et al. [9], many applications have incorporated this technique. Network coding is a technique that increases energy efficient and reduces network congestion by combining packets destined for distinct users. Afterwards, Li et al. prove that linear network coding is sufficient for the encoding functions [10]. COPE [11] is the first practical wireless network coding scheme designed to deal with inter-flow traffic in wireless networks. With opportunistic listening and opportunistic coding, COPE intends to exploit the shared nature of wireless medium. By combining what one neighbor wants with what other neighbors have, a router with COPE can transmit multiple packets to different neighbors in a single transmission. Experiments have shown that COPE can significantly improve network throughput. Hou et al. [12] propose AdapCode, a reliable data dissemination protocol that uses adaptive network coding to reduce broadcast traffic in the process of code updates. Packets on every node are coded by linear combination and decoded by Gaussian elimination. The core idea in AdapCode is to adaptively change the coding scheme according to the link quality. Fragouli et al. [13] proposed an instant primer on network coding, the author explain what network coding does and how it does it, and also discuss the implications of theoretical results on network coding for realistic settings and show how network coding can be used in practice. Kagi et al. [14] proposed an efficient and reliable packet transmission method by using multipath routing constructs from multiple node disjoint routes, and by applying network coding, which allows packet encoding at a relay node. For the problem of multipath under WSN environment, using network coding at the multipath can achieve the network capacity.

This paper proposes an Energy efficient that carefully couples Network coding and Multipath routing algorithm in Wireless Sensor Networks (ENM-WSN). It is typically proposed in order to increase the reliability of data transmission, and by applying network coding, which allows packet encoding at a relay node. We will also implement the performance difference between multipath routing based on energy Efficient and collision aware (EECA) [7], and network coding based reliable disjoint and braided multipath routing (NC-RMR ) protocol [17].

The rest of the paper is organized as follows: In Section 2, the paper briefly reviews the main theorem of network coding. Section 3 presents network coding scheme in WSN. Some simulating results are provided in Section 4. Finally, the paper concludes and future work in Section 5. 


\section{Main Theorem of Network Coding (NC)}

\subsection{Main Developments of Network Coding}

The value of the cut is the sum of the capacities of the edges on the cut. According to the multicast network problem statement, maximum flow from source to destinations in any network is equal to the size of min-cut [15]. As a result, there has been a great emphasis on linear network coding. For instance, Ho et al. [16] proposed a simple, practical code that achieves the min-cut of the network. They proposed that every node construct its linear code randomly and independently from all other nodes. This simple construction was shown to achieve capacity with probability exponentially approaching with increasing field size.

Network coding enhances energy efficient by reducing the number of packet transmissions. The basic concept of network coding, as applied to a flood-based application, can be explained using a simple scenario shown in figure 1. Intermediate nodes can re-encode and forward the linearly independent blocks on hand. The destination is able to decode once it receives a sufficient number of coded blocks.

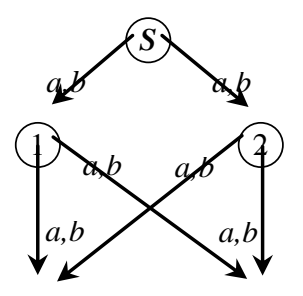

(3)
(4)

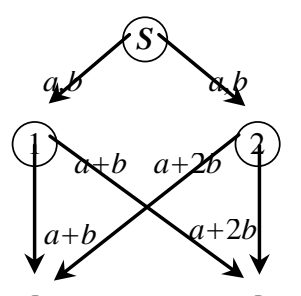

(3)
(4)

Figure 1. (a) Transmissions when Network Coding is not used (a Total of 6 Packet Transmissions); (b) Transmissions when Network Coding is used (4 Packet Transmissions)

Source node $S$ wants to flood two packets $a$ and $b$. As shown in figure 1(a), when network coding is not used, six packet transmissions are required to deliver the two packets to all nodes in the network, i.e., node 1, node 2, node 3, and node 4. As shown in figure 1(b), however, when network coding is used, only four packet transmissions are needed. This is because each of the two relays transmits only one coded packet. For network coding to work, receive node 3 , node 4 must be able to receive both coded packets, i.e., $(a+b)$ and $(a+2 b)$. Otherwise, they will be unable to decode the other packet received.

The binary symbol $(a+b)$ is a mathematical function of $a$ and $b$. Calculation of a function from received data is called coding. Showing the merit of mixed coding among multiple messages at an intermediate node, $(a+b)$ is called network coding (NC). In algebra, $(a+b)$ is called the binary sum of $a$ and $b$, interpreting in more general terms of linear algebra, the linear sum $(a+2 b)$ over the binary field. Thus, the calculation of $(a+b)$ is not only a form of coding but also a more restricted form of linear coding.

\subsection{Multipath Routing and Network Coding in WSN}

In WSN, nodes added or removed in ad hoc manner lead to network topology becoming unknown, and at the same, it is very difficult to predict packet loss, node and link failures. NC-based routing deals with the recoding of packets belonging to the same flow and is also known as intra-flow or intra-session coding. Protocols, i.e. network coding based reliable disjoint and braided multipath routing (NC-RMR) [17], and pipelined opportunistic routing 
(PipelineOR) [18] are related to NC-based routing and the use of NC reduces redundant data transmissions that lead to energy consumption reduction within wireless sensor networks. Akhtar et al. [19] propose two new energy efficient routing algorithms, namely, cooperativecost shortest path routing (CCSPR) and cooperation over shortest path non-cooperative routing (COSPNCR). Guo et al. [20] propose an efficient error-recovery scheme that carefully couples network coding and multiple paths.

$\mathrm{NC}$ allows each node to perform an operation, for example linear combinations of received data packets before forwarding on different transmission lines. Therefore, NC-aware routing is a special case of NC. NC-aware routing techniques take into account the availability of NC opportunities within a network during route selection for data transmission. Combining data packets from different flows along routes with more coding opportunities further improves network throughput.

\section{Network Coding Scheme (NCS)}

\subsection{Network Model}

This paper will discard such an unrealistic assumption, and develop a practicable model for network coding. We will propose the WSN model by using Poisson point process in $\mathbf{R}^{2}$. We consider a WSN where nodes are randomly distributed within a specified region $B \subset \mathbf{R}^{2}$. Let $\Phi_{0}$ be a Poisson point process in $\mathbf{R}^{2}$ with intensity $\lambda_{0}$ which characterizes the population of the nodes in the region $B$. Hence, there are $\lambda_{0}$ nodes in the WSN in average. Let $\Phi_{1}, \Phi_{2}, \cdots, \Phi_{N-1}$ be $N-12$-dimensional independent Poisson processes with intensities, $\lambda_{1}, \lambda_{2}, \cdots, \lambda_{N-1}$, which are also independent of $\Phi_{0} . N$ denotes the number of nodes. It is assumed that each node is equipped with an omnidirectional antenna and that its transmission power can be adjusted dynamically, to control its transmission range.

\subsection{Network Coding Model}

In our proposed method, network coding (NC), multiple link-disjoint routes are constructed. As shown in figure 1 , first, source node $\mathrm{S}$ sends data packets $a, b$ and $c$, encodes these packets into data 1 , data 2 and data3, and then sends them to destination node $D$. These packets will reach relays node 1 , node 2 and node 3 simultaneously because of the broadcast property of the acoustic channel. Node 1 receives data packets data1 and data 2 successfully and encodes them into packets data4 and data5 then forwards it to the next node. Node 2 receives data packets data 1 and data 3 successfully and encodes them into packets data6 and data7 then forwards it to the next node. Node 3 receives data packets data1, data2 and data3 successfully and encodes them into packets data8, data9 and data10 then forwards it to the next node. The destination node $D$ receives three encoded data packets data4, data6 and data10. When using a proper network coding scheme, the destination node $D$ can recover the three original packets with high probability.

Therefore, the number of transmissions does not need to be increased, and reliability can be improved because the encoded packet can be forwarded to destination node $D$.

\subsection{Network Coding Policy}

The network coding idea was introduced by Ahlswede et al. [9]. Usually, the routers or relay nodes just forward and duplicate the packets in the networks. However, network coding permits routers or relay nodes to encode the packets. In this paper, we use a linear network coding scheme [10]. 


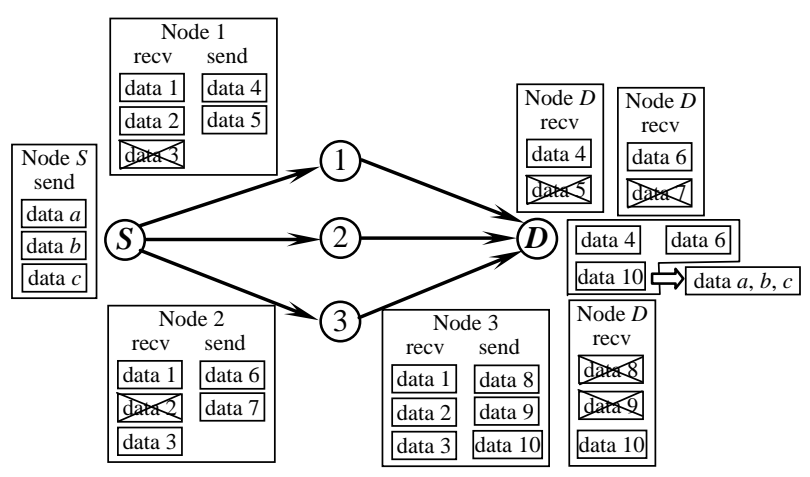

Figure 2. Sample Model using NC

Encoding: Packets from the source are divided into generations, with each generation containing $N$ packets. The linear network coding scheme is an encoding method such that coding vector $g_{i}=\left(g_{i 1}, g_{i 2}, \ldots, g_{i N}\right)$ is given, and input packet $M=\left(M_{1}\right.$, $M_{2}, \ldots, M_{N}$ ) is converted into output packet $P_{i}$ by the following expression [14].

$$
P_{i}=\sum_{j=1}^{N} g_{i j} M_{j}
$$

The destination node can decode input packets because the coding vector $G=\left(g_{1}\right.$, $\left.g_{2}, \ldots, g_{N}\right), g_{i j}$ is a randomly chosen coefficient from a Galois field such as $\mathrm{GF}\left(2^{8}\right)$, and output packet data $P=\left(P_{1}, P_{2}, \ldots, P_{N}\right)$ are obtained from the received packets, and an inverse matrix exists in $G$.

Decoding: Decoding at receiver node is performed by collecting packets of a given generation. The destination attempts to decode the incoming coded packets according to the following algorithm. These packets yield a system of linear equations that need to be solved to retrieve the original native packets. The decoding matrix represents the coefficient matrix of such a linear system. The destination uses Gauss-Jordan elimination to convert the decoding matrix to a reduced row echelon form and solve the linear system. Suppose a node has received $v$ encoded packets $X_{1}, X_{2}, \ldots, X_{v}$ belonging to a given generation, with $v \leq N$ while $g_{1}^{\prime}, g_{2}^{\prime}, \ldots, g^{\prime}$ represent the coding vectors corresponding to the encoded packets. The generic element of the decoding matrix $G$ is given by: $G_{i j}=g_{i j}$ where $i=1, \ldots, v$ and $j=1, \ldots, N$. Let us denote the rank of $G$ by $R$. When the matrix has full rank, i.e., $R=v=N$, for a given generation, then the node can solve the linear equations to retrieve all native packets belonging to that generation. In this case, the receiver can recover part of the source native packets belonging to the given generation. We finally observe that when a node receives a packet, it must check whether it is innovative or not, i.e., whether it increases the rank of the decoding matrix $G$. If not, the packet is dropped.

When the destination node $D$ receives $N$ packets with linearly independent encoding vectors, it recovers the original packets by matrix inversion [13].

\subsection{Energy Consumption Model}

In this section, we derive the energy efficient gains that can be achieved by using ENMWSN. We assume that the source node $S$, needs to transmit symbols to the destination node $D$. 
Without loss of generality, it is assumed that the symbol period for each symbol is normalized to one second.

Definition 1: The energy efficient gain of the network coding transmission over a non-network coding transmission, denoted by $G$, is defined as

$$
G=\frac{\varepsilon_{p t p}-\varepsilon_{n c p t p}}{\varepsilon_{p t p}}
$$

where $\varepsilon_{p t p}$ is the energy consumed by node $S$ in non-network coding transmitting the node $N$ packets towards node $D$ and $\varepsilon_{n c p t p}$ is the energy consumption if ENM-WSN is used to transmit the same $N$ packets.

Next, we derive $\varepsilon_{p t p}$ and $\varepsilon_{n c p t p}$. The energy consumption $\varepsilon_{p t p}$ can be written as

$$
\varepsilon_{p t p}=N * P_{p t p}
$$

where $P_{p t p}$ represents the average transmission power for point to point non-network coding transmission.

In order to transmit with a finite average power, we restrict a node to transmit only when the channel power gain is larger than a threshold. The transmitting node avoids transmission and declares an outage. Let $\rho_{u v}$ be the outage threshold for the channel between a source node $t_{u}$ and a receiving node $r_{v}$. Also, let $\delta$ indicate the probability of this outage, i.e., the probability that no transmission occurs between the source and the destination nodes. Then

$$
\delta=\operatorname{Pr}\left(\left|h_{u v}\right|^{2}<\rho_{u v}\right)
$$

The probability density function (PDF) of $\left|h_{u v}\right|^{2}$ is given by

$$
f\left(\left|h_{u v}\right|^{2}, 1 / \sigma_{u v}^{2}\right)=\frac{1}{\sigma_{u v}^{2}} \exp \left(-\frac{\left|h_{u v}\right|^{2}}{\sigma_{u v}^{2}}\right), \text { for }\left|h_{u v}\right|^{2} \geq 0
$$

Thus, the outage probability $\delta$ in (4) can be written as

$$
\delta=1-\exp \left(-\frac{\rho_{u v}}{\sigma_{u v}^{2}}\right)
$$

Assuming $\delta$ to be a fixed system parameter, one can find $\rho_{u v}$ from $(5,6)$ as

$$
\rho_{u v}=-\sigma_{u v}^{2} \ln (1-\delta)
$$

When the outage is not declared, the average transmit power in non-network coding transmitting a symbol from node 1 to node 2 can be expressed, the exponential distribution of the channel power gain, i.e., $\left|h_{12}\right|^{2}$, as follows

$$
P_{p t p}=-\beta d_{12}^{\alpha} \operatorname{Ei}(\ln (1-\delta))
$$

where $\beta=\gamma_{t h} P_{\eta}(4 \pi / \lambda)^{2} d_{f}^{2-\alpha}, \operatorname{Ei}(\delta)=\int_{-\infty}^{\delta} \frac{\exp (x)}{x} d x$. Then, using $P_{p t p}$ from (8) in (3), we arrive at

$$
\varepsilon_{p t p}=-N \beta d_{12}^{\alpha} \operatorname{Ei}(\ln (1-\delta))
$$


For ENM-WSN, node 1 transmits the first packet network coding towards node 2. The channel power gain between node 3 and node 2 is greater than the corresponding outage threshold $\rho_{32}$, then, the remaining packets are transmitted cooperatively using ENM-WSN.

$$
\begin{aligned}
\rho & =\operatorname{Pr}\left(\frac{P_{12}\left|h_{13}\right|^{2}}{P_{\eta}} \geq \gamma_{t h}\right) \times \operatorname{Pr}\left(\left|h_{32}\right|^{2} \geq \rho_{32}\right) \\
& =\frac{d_{12}^{\alpha}}{d_{12}^{\alpha}+d_{13}^{\alpha}}(1-\delta)^{2+d_{13}^{\alpha} / d_{12}^{\alpha}}
\end{aligned}
$$

The overall energy consumption can be written as

$$
\varepsilon_{n c p t p}=P_{p t p}+\left((N-1) P_{n c}\right)\left(1-\rho_{s}\right)+\left((N-1) P_{n 1}+(N-1) P_{n 2}\right) \rho_{s}
$$

where $P_{p t p}$ represents the average transmission power for the first symbol, given by (8), $P_{n c}$ represents the average transmission power of node 1 when ENM-WSN is not used while $P_{n 1}$ and $P_{n 2}$, respectively, represent the average transmission power of node 1 and node 3 when they network coding transmit using ENM-WSN.

\subsection{Analysis of Network Coding}

In this section, we provide some theoretical analysis on throughput efficiencies of the ENM-WSN techniques for multipath scenarios. Note that parts of this analysis have been introduced previously in [20]. The efficient of network coding relies on the quality of the underlying paths determined by a multipath routing algorithm. We next describe a property of a multipath under which network coding is efficient (in both error recovery and energy consumption).

Consider an arbitrary generation of $N$ packets. Under linear random coding, when a destination node receives at least $N$ packets in the generation, the probability that it recovers the $N$ original packets is high for a sufficiently large finite field.

We do not differentiate between the nodes in the same relay set. Let $p$ be the probability that a packet has bit error rate at receiver, $\beta_{i k}$ be the probability that a node in the $i$-th relay set receives $n$ packets (when $0 \leq k<N$ ) or at least $k$ packets (when $k=N$ ) from all nodes in the previous relay set, $1 \leq i \leq H$. Since the destination node is in the $H$ th relay set and the generation is arbitrary, we have $R=\beta_{H, N}$.

First, we assume that a packet loss occurs when there is at least error within a packet. Thus, the probability that this packet is transmitted successfully for $i$ hop(s), $p_{i}$, is

$$
p_{i}=(1-p)^{i}, 1 \leq i \leq H
$$

The successful delivery ratio, $R$, equals to the probability that the packet is transmitted successfully for $H$ hops. That is, $R=p_{H}=(1-p)^{H}$.

Let $\alpha_{i}$ be the probability that a node in the ith relay set receives packet $N$. Let $\alpha_{i, n}$ be the probability that $n$ nodes in the ith relay set receive packet $N, n=0, \ldots, N_{i}$. Assume that packet losses are independent. Then

$$
\begin{gathered}
\alpha_{i}= \begin{cases}1-p, & i=1 \\
\sum_{n=0}^{N_{i}-1} \alpha_{i-1, n}\left(1-p^{n}\right), & 2 \leq i \leq H\end{cases} \\
\alpha_{i, n}=\left(\begin{array}{l}
N_{i} \\
n
\end{array}\right) \alpha_{i}^{n}\left(1-\alpha_{i}\right)^{N_{i}-n}, \quad n=0, \cdots, N_{i}
\end{gathered}
$$


We next derive $\beta_{i k}, 1 \leq i \leq H, 0 \leq k<N$. The nodes in the first relay set receive packets from the source. Therefore

$$
\beta_{1, k}=\left\{\begin{array}{l}
(N k)(1-p)^{k} p^{N-k}, \quad 0 \leq k<N, \\
1-\sum_{j=0}^{N-1} \beta_{1, j}, \quad k=N .
\end{array}\right.
$$

where $N$ is the number of encoded packets from the source.

Let $\gamma_{i, j, k}$ denote the probability that a node in the $i$-th relay set receives $k$ packets from the $j$ th node in the previous relay set, $1 \leq i \leq H, 1 \leq j \leq N_{i-1}, 1 \leq k \leq N$. Since each relay transmits no more than $N$ packets, we have

$$
\begin{gathered}
\gamma_{i, j, k}=\sum_{n=k}^{N} \beta_{i-1, k}\left(\begin{array}{l}
n \\
k
\end{array}\right)(1-p)^{k} p^{n-k} \\
\beta_{i+1, k}=\sum_{k_{j}=0, \ldots, k} \prod_{j=1}^{N_{i}} \gamma_{i+1, j, k_{j}} \\
T^{i}=\left\{\begin{array}{l}
N^{\prime} / N, \\
\frac{N_{i-1}}{N} \sum_{k=0}^{N} k \beta_{i-1, k}, \quad 2 \leq i \leq H
\end{array}\right.
\end{gathered}
$$

\section{Simulation Experiments}

In this section, we evaluate the performance of the proposed algorithms against the existing algorithms through the simulations. We consider a network of randomly distributed nodes within a specified square area. The source and the destination nodes are located in the diagonal corners of the network.

\subsection{Simulation Model and Performance Metrics}

To conduct the simulation studies, we have used randomly generated networks on which the algorithms were executed [21]. This ensures that the simulation results are independent of the characteristics of any particular network topology.

Energy model: We assumed that the energy required to send a message to a node at a distance 1 from a source is proportional to the square of 1 . Its initial value corresponds to the node energy level at the beginning of the simulation. We also assumed that the energy required to receive a message is the sum of the fixed cost of powering the antenna plus the fixed processing cost. When the node energy level goes down to zero, the node dies out, that is no more packets can be received or transmitted by the node. The initial energy of each node battery is $30 \mathrm{~J}$ in the reference scenario.

So, a propagation model is used to determine the Signal to Noise Ratio (SNR) at the receiving node. SNR is defined as the ratio of power of the receiving signal to the noise power at the receiver.

To effectively evaluate ENM-WSN's performance, we compare it with other famous multipath routing protocols, EECA [7], and NC-RMR [17] for cost to control information, average link-connect time, the success rate to find the path and the feature of data transmission. Table 1 lists the simulation parameters which are used as default values unless otherwise specified. 
Table 1. Simulation Parameters

\begin{tabular}{|c|c||c|c|}
\hline Number of nodes & 100 & Correct receive threshold & $3.652 \times 10^{-10} \mathrm{~W}$ \\
\hline Terrain range & $1000 \mathrm{~m} \times 1000 \mathrm{~m}$ & Threshold to avoid collisions & $1.559 \times 10^{-11} \mathrm{~W}$ \\
\hline Transmission range & $250 \mathrm{~m}$ & Channel bandwidth & $1-3 \mathrm{Mbps}$ \\
\hline Average node degree & $3-5$ & Links delay & $20-200 \mathrm{~ms}$ \\
\hline Simulation time & $600 \mathrm{~S}$ & Traffic type & CBR \\
\hline Maximum transmit power $T_{t}^{\max }$ & $0.282 \mathrm{~W}$ & Node pause time & $10 \mathrm{~S}$ \\
\hline Initial node energy & $30 \mathrm{~J}$ & Examined routing protocol & EECA and NC-RMR \\
\hline
\end{tabular}

We will compare the performance of three multipath routing methods under the same energy model and communication models. Performance metrics we have used in our experiments are energy consumption, packet delivery ratio (PDR), and network lifetime.

1) Energy consumption: Energy consumption is measured at the radio layer during the simulation based on the specification of IEEE 802.11- WLAN. The power consumption varies from 0.013 Watt in a low-power sleep state to $0.83,1.0$, and 1.4 Watt in idle listening, receiving, and transmitting states, respectively,

2) Packet delivery ratio (PDR): Defined as the ratio of the average number of data packets received by the destination node to the number of data packets transmitted by the source, and is obtained as follows.

Packet delivery ratio $=($ No. of packets delivered $) /($ No. of packets sent $)$

The number of delivered data packets is the summation of total numbers of delivered data packets received by each node. The number of sent data packets is the summation of total numbers of sent data packets of each node. The packet delivery ratio shows the transmission Efficient of the network with the given protocol.

3) Network lifetime: It is defined as the active period of the network nodes that have been able to process and transmit data until a node fails due to exhaustion of its battery, resulting in the partition of the network.

\subsection{Simulation Results}

The results of the simulation are positive with respect to performance. We use the Network Simulator version 2 (NS-2) [22] to evaluate the NC-MR protocols. NS-2 is a discrete event simulator targeted at networking research. NS-2 provides substantial support for simulation of TCP, routing, and multipath protocols over wired and wireless networks.

In the sequel, we consider the impact of the bit error rate (BER) on the energy saving gain expression in (4). The bit error rate is in the range of $10^{-4}$ to $10^{-3}$ to account for potentially high loss rate in WSN. For network coding, a generation contains three packets (e.g., $M=3$ ). Figure 3 compares the energy consumption of the ENM-WSN algorithm with EECA and NCRMR. From our experiments, it appears that network coding outperforms the other schemes: it achieves the lowest energy consumption for most of the bit error rates. It can be observed that energy efficiency is greatly improved by employing network coding.

Figure 4 compares the packet delivery ratio of the ENM-WSN algorithm with EECA and NC-RMR. The delivery ratio presents the ratio of the number of packets received by multipath receivers versus the number of data packets supposed to be received. For all kinds of traffic load, all schemes performance is affected by the increasing bit error rate. The 
proposed ENM-WSN has the better packet delivery ratio than that of EECA and NC-RMR, because both are using a traditional multipath structure, which can bring the network codingbased multipath route and energy Efficient for the packets to deliver.

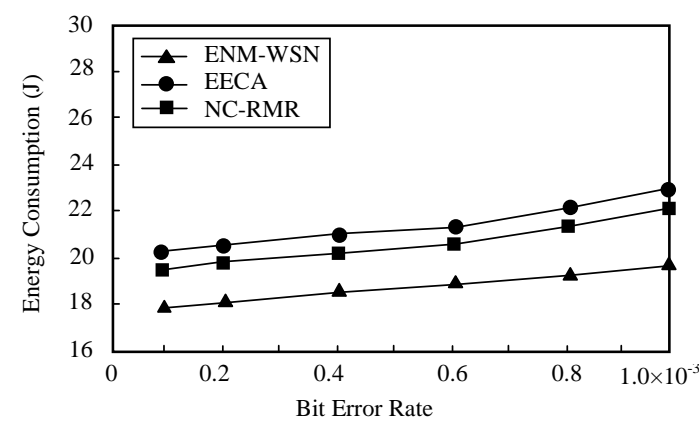

Figure 3. Comparison of Energy Consumption

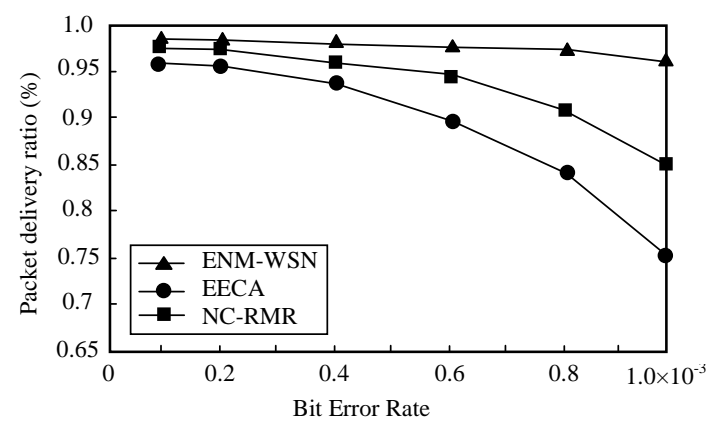

Figure 4. Comparison of Packet Delivery Rate

For maximizing the network life time, we consider interference free communication model in the sense that at each time instance only one node may transmit. Figure 5 show that the network lifetime has a decreasing trend as the bit error rate increases. Under the entire max bit error rate, ENM-WSN gives much longer lifetime than EECA and NC-RMR protocol does. ENM-WSN gets nearly 10-30\% higher than EECA and NC-RMR in lifetime. We can also observe that the network lifetime with multipath routing degrades more gracefully than with other routing protocols does when the bit error rate increases.

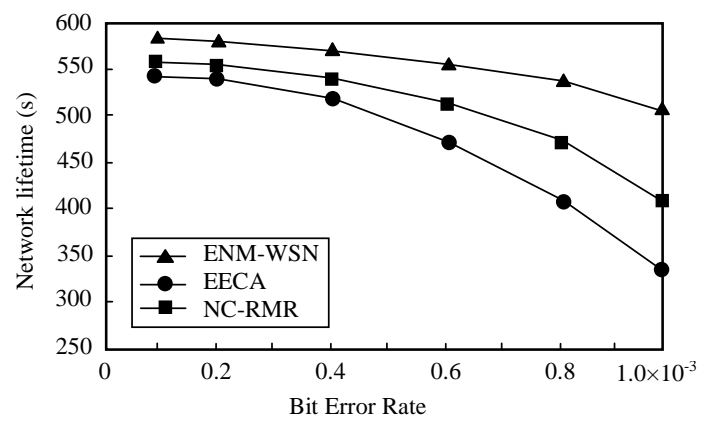

Figure 5. Comparison of network lifetime

\section{Conclusion}

Network coding and multipath routing are two popular techniques for saving energy in WSN. In this paper, we propose an energy Efficient that carefully couples network coding and multipath. Then we analytically study the performance of our scheme along with several other error recovery schemes. Last, we evaluate the performance of various schemes through simulation. The simulation results confirm the analytical study that our scheme is efficient in energy consumption, packet delivery ratio, and network lifetime in WSN.

In terms of future work, we would definitely consider optimizing the timeout values and other parameters used in NC-MR for further evaluation via simulation. Use of the nodedisjoint paths in parallel to improve the performance and increase the network utilization, is left as our future work. 


\section{Acknowledgements}

This work is supported by The Young and Middle-aged Elitists' Scientific and Technological Innovation Team Project of the Institutions of Higher Education in Hubei Province (No. T200902), Natural Science Foundation of Hubei Province of China (No. 2013CFB035, 2013CFB309), Key Scientific Research Project of Hubei Education Department (No. B20129205), Key Project of Hubei Province Education Science during the 12th Five-Year (2012A042, 2013B128).

\section{References}

[1] B. L. Sun, C. Gui, and Y. Song, "Energy Entropy-based Clusterhead Selection Algorithm for Ad Hoc Networks", International Journal of Advancements in Computing Technology (IJACT), vol. 4, no. 1, (2012), pp. 207-214.

[2] B. L. Sun, C. Gui, and Y. Song, "Energy Entropy On-Demand Multipath Routing Protocol for Mobile Ad Hoc Networks", China Communications, vol. 8, no. 7, (2011), pp. 75-83.

[3] B. L. Sun, X. C. Lu, C. Gui, Y. Song, and H. Chen, "Network Coding-Based On-Demand Multipath Routing in MANET", Proceedings of 26th IEEE International Parallel \& Distributed Processing Symposium (IPDPS), Shanghai, China, May 21-25, (2012), pp. 1514-1518.

[4] B. L. Sun, Y. Song, C. Gui and M. Luo, "Network Coding-Based Priority-Packet Scheduler Multi-path Routing in MANET using Fuzzy Controllers", International Journal of Future Generation Communication and Networking, vol.7, no.2, (2014), pp.137-148

[5] M. K. Anantapalli, and W. Li, "Multipath multihop routing analysis in mobile ad hoc networks", Wireless Network, vol. 16, no. 1, (2010), pp. 79-94.

[6] M. Leinonen, J. Karjalainen and M. Juntti, "Distributed power and routing optimization in single-sink data gathering wireless sensor networks", Proceedings of 9th European Signal Processing Conference (EUSIPCO 2011), Barcelona, Spain, (2011) August 29-September 2, pp. 407-411.

[7] Z. J. Wang, E. Bulut and B. K. Szymanski, "Energy Efficient Collision Aware Multipath Routing for Wireless Sensor Networks", Proceedings of IEEE ICC (2009), Dresden, Germany, June, 14-18, (2009), pp. $1-5$.

[8] J. Yoo, L. Yan, S. Lee, Y. Kim, and H.-J. Yoo, “A $5.2 \mathrm{~mW}$ self-configured wearable body sensor network controller and a $12 \mu \mathrm{W}$ wirelessly powered sensor for a continuous health monitoring system", IEEE Journal of Solid-State Circuits, vol. 45, no. 1, (2010), pp. 178-188.

[9] R. Ahlswede, N. Cai, S.-Y.R. Li, and R. W. Yeung, "Network information flow", IEEE Transactions on Information Theory, vol. 46, no. 4, (2000), pp. 1204-1216.

[10] S.-Y.R. Li, R.W. Yeung, and N. Cai, "Linear network coding", IEEE Transactions on Information Theory, vol. 49, no. 2, (2003), pp. 371-381.

[11] S. Katti, H. Rahul and W. Hu, et al. "XORs in The Air: Practical Wireless Network Coding", IEEE/ACM Transactions on Networking, vol. 16, no. 3, (2008), pp. 497-510.

[12] I.-H. Hou, Y.-E. Tsai, T. F. Abdelzaher, and I. Gupta, "AdapCode: Adaptive Network Coding for Code Updates in Wireless Sensor Networks", Proceedings of IEEE INFOCOM 2008, Phoenix, AZ, USA, (2008) April 15-17, pp. 2189-2197.

[13] C. Fragouli, J. L. Boudec, and J. Widmer, "Network coding: An instant primer", ACM SIGCOMM Computer Communication Review, vol. 36, no. 1, (2006), pp. 63-68.

[14] T. Kagi and O. Takahashi, "Efficient Reliable Data Transmission Using Network Coding in MANET Multipath Routing Environment", Proceedings of 12th International Conference on Knowledge-Based Intelligent Information and Engineering Systems (KES 2008), Zagreb, Croatia, (2008) September 3-5, pp. $183-192$.

[15] G. Dantzig, and D. R. Fulkerson, "On the max flow min cut theorem of networks", Stanford University Press, (2003).

[16] T. Ho, M. Médard, R. Kötter, M. Effros, J. Shi, and D. R. Karger, "A random linear coding approach to multicast”, IEEE Transactions on Information Theory, vol. 52, no. 10, (2006), pp. 4413-4430.

[17] Y. Yang, C. Zhong, Y. Sun, and J. Yang, "Network coding based reliable disjoint and braided multipath routing for sensor networks", Journal of Network and Computer Applications, vol. 33, no. 4, (2010), pp. 42232.

[18] Y. J. Lin, C. C. Huang, and J. L. Huang, "PipelineOR: a pipelined opportunistic routing protocol with network coding in wireless mesh networks", Proceedings of the 71st IEEE vehicular technology conference VTC'10), Taipei, Taiwan, (2010) May 16-19, pp. 1-5. 
[19] A. M. Akhtar, M. R. Nakhai and A. H. Aghvami, "On the Use of Cooperative Physical Layer Network Coding for Energy Efficient Routing”, IEEE Transactions on Communications, vol. 61, no. 4, (2013), pp. 1498-1509.

[20] Z. Guo, B. Wang, P. Xie, W. Zeng, and J. H. Cui, "Efficient error recovery with network coding in underwater sensor networks", Ad Hoc Networks, vol. 7, no. 4, (2009), pp. 791-802.

[21] B. Waxman, "Routing of Multipoint Connections", IEEE Journal on Selected Areas in Communications, vol. 6, no. 9, (1988), pp. 1617-1622.

[22] The Network Simulator - NS-2: http://www.isi.edu/ nsnam/ns/.

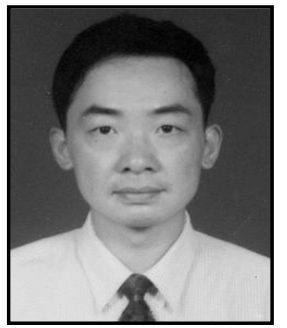

Chao Gui received his M.S. degree from Wuhan University, China in 1989. He is currently a professor in computer science and technology of Hubei University of Economics, China. His research interests include wireless communication, performance analysis and analytical modeling. He has published over 60 research papers. E-mail: gui_chao@126.com.

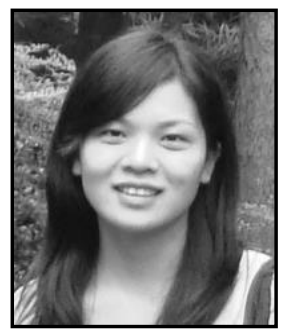

Hua Chen received his M.S. degree from Wuhan University, China in 2005. She is currently an associate professor of mathematics and computer science of Wuhan Textile University, China. Her research interests include algorithm, wireless communication, performance analysis, and network model. She has published over 30 research papers. E-mail: qiuchen_1022@163.com.

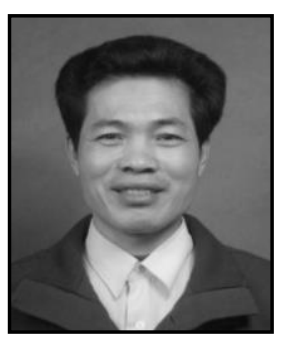

Baolin Sun received his $\mathrm{Ph} . \mathrm{D}$. degree from Wuhan University of Technology, China in 2006. He is currently a professor in computer science and technology of Hubei University of Economics, China. His research interests include multipath routing, parallel and distributed computing, network optimization and ad hoc networks. He has published over 120 journal and conference papers and has author of four books in the above areas. E-mail: blsun@163.com.

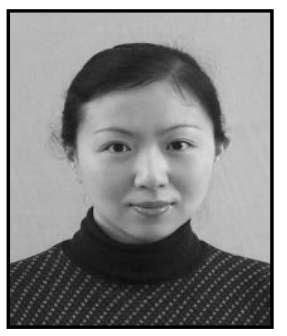

Ying Song received her Ph.D. degree from Wuhan University, China in 2011. She is currently an associate professor in computer science and technology of Hubei University of Economics, China. Her research interests include wireless communication, mesh networks and network protocol. She has published over 30 research papers. E-mail: prisong@163.com. 